\title{
Effect of control-beam polarization and power on optical time-domain demultiplexing in a new nonlinear optical loop mirror design
}

\author{
Drahomír Grendár \\ T-Com \\ Karadžičova 10 \\ 82513 Bratislava \\ Slovakia
}

Olivier Pottiez

Centro de Investigaciones en Óptica

Loma del Bosque 115

Col. Lomas del Campestre

León Gto. 37150

Mexico

\author{
Milan Dado \\ University of Žilina \\ Faculty of Electrical Engineering \\ Department of Telecommunications \\ and Multimedia \\ Univerzitná 1 \\ 01026 Žilina \\ Slovakia
}

\author{
Jarmila Müllerová \\ University of Žilina \\ Faculty of Electrical Engineering \\ Department of Engineering Fundamentals \\ kpt. J. Nálepku 1390 \\ 03101 Liptovský Mikuláš \\ Slovakia \\ E-mail: mullerova@Im.uniza.sk \\ Jozef Dubovan \\ University of Žilina \\ Faculty of Electrical Engineering \\ Department of Telecommunications \\ and Multimedia \\ Univerzitná 1 \\ 01026 Žilina \\ Slovakia
}

\begin{abstract}
A new scheme of a control-beam-driven nonlinear optical loop mirror (NOLM) with a birefringent twisted fiber and a symmetrical coupler designed for optical time division demultiplexing (OTDM) is analyzed. The theoretical model of the proposed NOLM scheme considers the evolution of polarization states of data and control beams and the mutual interactions of the data and control beams due to the cross-phase modulation (XPM). Attention is given to the optical switching commanded by the control-beam power and by the manipulation of nonlinear polarization rotation of the data and control beam. The simulations of NOLM transmissions demonstrate that the cross talk between demultiplexed and nondemultiplexed beams as an important parameter for optical switching by the presented NOLM can be significantly reduced. The results show that the device can be of interest for all-optical signal manipulations in optical communication networks. (๑) 2009 Society of Photo-Optical Instrumentation Engineers. [DOI: 10.1117/1.3125427]
\end{abstract}

Subject terms: nonlinear optical loop mirror (NOLM); optical switching; demultiplexing; polarization; control beam.

Paper 080960R received Dec. 9, 2008; revised manuscript received Mar. 3, 2009; accepted for publication Mar. 8, 2009; published online May 5, 2009.

\section{Introduction}

One of the promising candidates for optical processing of signals like ultra-fast switching and demultiplexing of future high-bit-rate optical time division multiplexing (OTDM) signals is the nonlinear optical loop mirror (NOLM) switch. ${ }^{1-3}$ There is a potential for demultiplexing data at speeds beyond $1 \mathrm{Tbit} / \mathrm{s} .{ }^{4-6}$ The nonlinear transmission characteristics of the NOLM can be also utilized for other all-optical applications such as filtering, logical operations, ${ }^{7,8}$ all-optical active mode locking, ${ }^{9}$ passive

0091-3286/2009/\$25.00 @ 2009 SPIE mode locking, ${ }^{10}$ pedestal suppression, ${ }^{11}$ amplitude regularization of optical pulse trains, ${ }^{12}$ or regeneration of ultrafast data streams. ${ }^{13}$

The NOLM is a fiber loop with a nonlinear element constituting the Sagnac interferometer. All-optical switching speeds of $\sim \mathrm{Tbit} / \mathrm{s}$ are possible, as the NOLM is based on the ultra-fast Kerr effect in the optical fiber. ${ }^{14}$ There are two principal schemes of all-optical NOLM switches: a data-beam-driven switch and a control-beam-driven NOLM. $^{2}$ In both, the switching effect is based on the difference in the nonlinear phase shift between two counterpropagating signals that causes the power-dependent transmission characteristics of the NOLM.

In the control-beam-driven NOLM, the input data beam 
to be demultiplexed or switched is divided into two counterpropagating signals $(\mathrm{CW}$-clockwise; $\mathrm{CCW}$ counterclockwise) by a symmetrical or an asymmetrical coupler. The control signal is coupled into the loop through the control port and propagates in the loop in only one direction $(\mathrm{CW})$. The switching is commanded by the power of the control signal. In the absence of the control signal, the device acts as a mirror, and the data signal is returned to the input port. The data and control signals must be thoroughly synchronized so that no additional phase shift caused by data signal time jitter occurs. ${ }^{15}$

The control-beam-driven switching in a conventional NOLM is based on the cross-phase modulation (XPM) ${ }^{2,3}$ or the self-phase modulation (SPM $)^{16-18}$ in both polarizationmaintaining and nonpolarization-maintaining fibers. The key point of the scheme proposed by Kuzin et al. ${ }^{16-18}$ relies on the nonlinear polarization rotation thanks to the use of a twisted nonlinear fiber and a quarter-wave plate inserted in the loop. This offers more degrees of freedom for the adjustment of the transmission characteristics. ${ }^{19,20}$

The possibility to adjust the control-beam power to reach the required value of the NOLM transmission is of particular interest in the OTDM applications. In addition, the greater flexibility of the control-beam-driven NOLM for OTDM systems results from the exploitation of polarization effects. The degree of interaction between data and control beams due to XPM depends on the relative orientation of the beams' polarizations. Then, through the adjustment of this relative orientation, the transmission and therefore also the switching properties can be tuned.

This paper studies the performance of a new NOLM scheme modified in comparison with the Kuzin's NOLM design. ${ }^{16-18}$ We analyze a control-beam-driven NOLM using a symmetrical data input coupler in which the switching properties are achieved by control-beam power and the management of the nonlinear polarization rotation between data and control beams only. The transmissions of the NOLM scheme without a quarter-wave plate are simulated based on the theoretical model presented in the Sec. 2.

Particular attention is given to the cross-talk reduction achieved by the management of the relative polarization orientations between co- and counterpropagating data and control beams. In general, the switching by a control-beamdriven NOLM results from the interaction of the control beam with the fraction of the copropagating data beam through XPM. However, when the average power of the control beam is large enough, the interaction between the control beam and counterpropagating data beam also occurs and may become significant. Then, the low-value transmission depends on the average control-beam power and may become nonzero, which represents the cross talk between demultiplexed and nondemultiplexed OTDM channels. This paper reports that in the presented scheme of the NOLM, a significant cross talk reduction can be achieved.

\section{Theoretical Model of a Control-Beam-Driven NOLM}

In this section, a theoretical model of the presented controlbeam-driven NOLM scheme is described. In our NOLM scheme, a birefringent twisted fiber and a symmetrical coupler are supposed. In such an NOLM, the beam propagation is influenced by both birefringence and optical activity due to the fiber twist. Therefore, the NOLM operation depends strongly on the input polarization. The presented theoretical model gives an idea of the behavior of the NOLM scheme considering the evolution of polarization states of data and control beams. Moreover, the mutual interactions of the data and control beam due to XPM must be taken into consideration. The beam attenuation, the dispersion of the fiber, and the pulse dynamics are not considered in this model.

\subsection{Polarization Equations}

This subsection considers the polarization state evolution of propagating beams in the steady-state approximation. ${ }^{21,22}$ Our approximation neglects the fiber dispersion and is valid when the pulse widths are sufficiently long to yield a dispersion length longer than the fiber length. ${ }^{16}$ The next differential equations describe the polarization states of beams propagating in a birefringent twisted fiber along the $z$ axis in the circular polarization basis:

$\frac{\partial C^{+}}{\partial z}=i \frac{h q}{2 n} C^{+}+i k \exp (2 i q z) C^{-}$,

$\frac{\partial C^{-}}{\partial z}=-i \frac{h q}{2 n} C^{-}+i k \exp (-2 i q z) C^{+}$,

where $C^{+}$and $C^{-}$are complex amplitudes of right $(\mathrm{CW})$ and left $(\mathrm{CCW})$ circular polarization components normalized to the square root of the beam power, $h q$ is the circular birefringence ( $q$ is the fiber twist rate, and $h \approx 0.13 \div 0.16$ for silica fiber is a parameter related to the circular birefringence and the fiber twist), $n$ is the refractive index, and $k$ $=\pi \Delta n / \lambda$ is a parameter related to the linear birefringence $\Delta n$ ( $\lambda$ is the wavelength). The $k$ parameter is connected with the so-called fiber beat length $L_{b}$ as $L_{b}=\pi / k$.

Amplitudes of polarized beams propagating along the loop can be defined by the normalized Stokes parameter $A$ (in the circular polarization basis) and by the polarization angle $\psi$. For the propagating beams in the circular polarization basis, the following is valid:

$A=\left|C^{+}\right|^{2}-\left|C^{-}\right|^{2} \quad 1=\left|C^{+}\right|^{2}+\left|C^{-}\right|^{2}$,

$C^{+}=\left(\frac{1+A}{2}\right)^{1 / 2} e^{i \psi} \quad C^{-}=\left(\frac{1-A}{2}\right)^{1 / 2} e^{-i \psi}$,

where $\psi$ defines the direction of the ellipse major axis to the chosen direction.

Now let us transform Eqs. (1) and (2), into the often used eigenmode polarization basis. ${ }^{16}$ We transform complex amplitudes $C^{+}$and $C^{-}$into polarization eigenmodes $S^{+}$ and $S^{-}$.

$S^{+}=\cos \alpha \cdot C^{+}+\sin \alpha \cdot C^{-} \quad C^{+}=\cos \alpha \cdot S^{+}-\sin \alpha \cdot S^{-}$, 
$S^{-}=-\sin \alpha \cdot C^{+}+\cos \alpha \cdot C^{-} \quad C^{-}=\sin \alpha \cdot S^{+}+\cos \alpha \cdot S^{-}$,

where $\alpha=\arctan (k / \gamma) / 2$. The parameter $\gamma=(h / 2 n-1) q$ expresses the ratio of the fiber twist to the birefringence.

The normalized beam power $P$ can be expressed from Eqs. (3)-(6) using $C^{+2}+C^{-2}=S^{+2}+S^{-2}$ :

$$
\begin{aligned}
P= & {\left[\left(\frac{1+A}{2}\right)^{1 / 2} e^{i \psi} \cos \alpha+\left(\frac{1-A}{2}\right)^{1 / 2} e^{-i \psi} \sin \alpha\right] S^{+} } \\
& +\left[-\left(\frac{1+A}{2}\right)^{1 / 2} e^{i \psi} \sin \alpha+\left(\frac{1-A}{2}\right)^{1 / 2} e^{-i \psi} \sin \alpha\right] S^{-} .
\end{aligned}
$$

Deriving equations of polarization eigenmodes (5) and (6), applying them on Eqs. (1) and (2), and substituting for $C^{+}$ and $C^{-}$, we obtain the following linear propagation equations:

$$
\begin{aligned}
\frac{\partial S^{+}}{\partial z}= & i\left(\frac{h q}{2 n} \cos 2 \alpha+k \sin 2 \alpha \cos 2 q z\right) S^{+}+[-k \sin 2 q z \\
& \left.+i\left(-\frac{h q}{2 n} \sin 2 \alpha+k \cos 2 \alpha \cos 2 q z\right)\right] S^{-}, \\
\frac{\partial S^{-}}{\partial z}= & {\left[k \sin 2 q z+i\left(-\frac{h q}{2 n} \sin 2 \alpha+k \cos 2 \alpha \cos 2 q z\right)\right] S^{+} } \\
& +i\left(\frac{h q}{2 n} \cos 2 \alpha-k \sin 2 \alpha \cos 2 q z\right) S^{-} .
\end{aligned}
$$

\subsection{XPM between Polarized Eigenmodes}

In a birefringent fiber, the state of polarization of two generally elliptically polarized waves changes with propagation. The orthogonally polarized components of each wave are then mutually coupled through XPM. The resulting set of four coupled Schrödinger equations neglecting the beam attenuation and the dispersion of the fiber is based on Ref. 23 and 24. The following nonlinear equations representing the propagation of the two polarized components of both the data and control beam expressed in terms of polarization eigenmodes are:

$$
\frac{\partial S_{1}^{+}}{\partial z}=i\left[\gamma_{1} P_{I N 1}\left(\left|S_{1}^{+}\right|^{2}+B\left|S_{1}^{-}\right|^{2}\right)+\gamma_{1} P_{I N 2}\left(2\left|S_{2}^{+}\right|^{2}+B\left|S_{2}^{-}\right|^{2}\right)\right] S_{1}^{+},
$$

$$
\frac{\partial S_{1}^{-}}{\partial z}=i\left[\gamma_{1} P_{I N 1}\left(B\left|S_{1}^{+}\right|^{2}+\left|S_{1}^{-}\right|^{2}\right)+\gamma_{1} P_{I N 2}\left(B\left|S_{2}^{+}\right|^{2}+2\left|S_{2}^{-}\right|^{2}\right)\right] S_{1}^{-},
$$

$$
\frac{\partial S_{2}^{+}}{\partial z}=i\left[\gamma_{2} P_{I N 1}\left(2\left|S_{1}^{+}\right|^{2}+B\left|S_{1}^{-}\right|^{2}\right)+\gamma_{2} P_{I N 2}\left(\left|S_{2}^{+}\right|^{2}+B\left|S_{2}^{-}\right|^{2}\right)\right] S_{2}^{+},
$$

$\frac{\partial S_{2}^{-}}{\partial z}=i\left[\gamma_{2} P_{I N 1}\left(B\left|S_{1}^{+}\right|^{2}+2\left|S_{1}^{-}\right|^{2}\right)+\gamma_{2} P_{I N 2}\left(B\left|S_{2}^{+}\right|^{2}+\left|S_{2}^{-}\right|^{2}\right)\right] S_{2}^{-}$,

where the amplitudes of the polarized components of the data $\left(S_{1}^{+}, S_{1}^{-}\right)$and control $\left(S_{2}^{+}, S_{2}^{-}\right)$beam slowly vary through XPM, and $P_{I N 1,2}$ stand for input peak powers of data (1) and control (2) beam. The parameter $B=2 / 3$ for a linearly birefringent fiber, ${ }^{23}$ and the nonlinear parameter $\gamma_{j}$ $=n_{2} \omega_{j} / c A_{\text {eff }}, j=1,2$, where $n_{2}$ is the nonlinear refractive index (the Kerr coefficient), $c$ is the speed of light, $A_{\text {eff }}$ the effective modal area, and $\omega_{j}$ is the beam circular frequency. ${ }^{23}$

\subsection{The Complete Set of Propagation Equations}

Equations (8) and (9) describe linear effects of the propagation of two polarized beams along the birefringent twisted fiber loop, and nonlinear Eqs. (10)-(13) represent their mutual interactions due to XPM. By adding linear and nonlinear propagation equations together, a complete characteristic of the propagation of two beams (in terms of NOLM - data and control beams) along the fiber loop and their mutual interactions during this propagation can be obtained.

The data- and control-beam amplitudes in terms of polarization eigenmodes satisfy the following coupled equations. (Subscripts 1 and 2 are related to the data and control beam, respectively.)

$$
\begin{aligned}
\frac{\partial S_{1}^{+}}{\partial z}= & i\left[\frac{h q}{2 n} \cos 2 \alpha_{1}+k_{1} \sin 2 \alpha_{1} \cos 2 q z\right. \\
& \left.+\gamma_{1} P_{I N 1}\left(\left|S_{1}^{+}\right|^{2}+B\left|S_{1}^{-}\right|^{2}\right)+\gamma_{1} P_{I N 2}\left(2\left|S_{2}^{+}\right|^{2}+B\left|S_{2}^{-}\right|^{2}\right)\right] S_{1}^{+} \\
& +\left[-k_{1} \sin 2 q z+i\left(-\frac{h q}{2 n} \sin 2 \alpha_{1}\right.\right. \\
& \left.\left.+k_{1} \cos 2 \alpha_{1} \cos 2 q z\right)\right] S_{1}^{-}
\end{aligned}
$$

$$
\begin{aligned}
\frac{\partial S_{1}^{-}}{\partial z}= & i\left[\frac{h q}{2 n} \cos 2 \alpha_{1}-k_{1} \sin 2 \alpha_{1} \cos 2 q z+\gamma_{1} P_{I N 1}\left(B\left|S_{1}^{+}\right|^{2}\right.\right. \\
& \left.\left.+\left|S_{1}^{-}\right|^{2}\right)+\gamma_{1} P_{I N 2}\left(B\left|S_{2}^{+}\right|^{2}+2\left|S_{2}^{-}\right|^{2}\right)\right] S_{1}^{-}+\left[k_{1} \sin 2 q z\right. \\
& \left.+i\left(-\frac{h q}{2 n} \sin 2 \alpha_{1}+k_{1} \cos 2 \alpha_{1} \cos 2 q z\right)\right] S_{1}^{+}
\end{aligned}
$$

$$
\begin{aligned}
\frac{\partial S_{2}^{+}}{\partial z}= & i\left[\frac{h q}{2 n} \cos 2 \alpha_{2}+k_{2} \sin 2 \alpha_{2} \cos 2 q z+\gamma_{2} P_{I N 1}\left(2\left|S_{1}^{+}\right|^{2}\right.\right. \\
& \left.\left.+B\left|S_{1}^{-}\right|^{2}\right)+\gamma_{2} P_{I N 2}\left(\left|S_{2}^{+}\right|^{2}+B\left|S_{2}^{-}\right|^{2}\right)\right] S_{2}^{+}+\left[-k_{2} \sin 2 q z\right. \\
& \left.+i\left(-\frac{h q}{2 n} \sin 2 \alpha_{2}+k_{2} \cos 2 \alpha_{2} \cos 2 q z\right)\right] S_{2}^{-}
\end{aligned}
$$




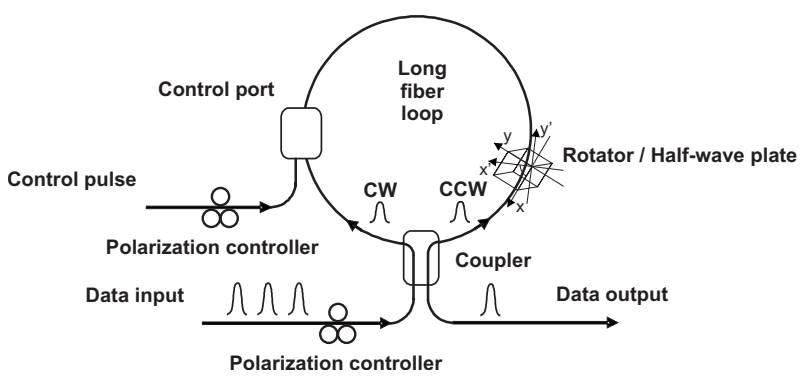

Fig. 1 The NOLM scheme.

$$
\begin{aligned}
\frac{\partial S_{2}^{-}}{\partial z}= & i\left[\frac{h q}{2 n} \cos 2 \alpha_{2}-k_{2} \sin 2 \alpha_{2} \cos 2 q z+\gamma_{2} P_{I N 1}\left(B\left|S_{1}^{+}\right|^{2}\right.\right. \\
& \left.\left.+2\left|S_{1}^{-}\right|^{2}\right)+\gamma_{2} P_{I N 2}\left(B\left|S_{2}^{+}\right|^{2}+\left|S_{2}^{-}\right|^{2}\right)\right] S_{2}^{-}+\left[k_{2} \sin 2 q z\right. \\
& \left.+i\left(-\frac{h q}{2 n} \sin 2 \alpha_{2}+k_{2} \cos 2 \alpha_{2} \cos 2 q z\right)\right] S_{2}^{+} .
\end{aligned}
$$

Equations (14)-(17) have been used as the basis in the output data- and control-beam power simulations. The NOLM transmission $T$ has been defined as $T \equiv P_{O U T} / P_{I N 1}$, where $P_{\text {OUT }}$ is the total output power of both polarization components of data beams, $P_{I N 1}$ is the data input power consisting of both $\mathrm{CW}$ and $\mathrm{CCW}$ components. The NOLM transmission is considered to be the NOLM transfer characteristics to study the demultiplexing performance. The polarization and control-beam power-dependent switching operation and cross talk have been the main objective of the calculations and discussions.

\section{Transmissions of a NOLM Driven by Control- Beam Polarization and Power}

According to the theoretical model, the control-beam peak power and polarization-dependent NOLM transmissions originate from nonlinear phase shift between polarized counterpropagating beams coupled by XPM in a birefringent fiber. In order to analyze the influence of the controlbeam properties on the NOLM switching, we study numerical results of NOLM transmissions related to the presented theoretical model.

The NOLM scheme (Fig. 1) consists of the conventional nontwisted $(q=0)$ fiber (ITU-T G.652) with length of $300 \mathrm{~m}$. The symmetrical $3-\mathrm{dB}$ data input coupler is used. The data- and control-beam wavelengths are $1550 \mathrm{~nm}$ and $1555 \mathrm{~nm}$, respectively. For all simulations, the following parameters representing the NOLM properties were used: the silica fiber constant $h=0.141$, the fiber beat length $L_{b}$ $=15 \mathrm{~m}$ (Ref. 25), the fiber refractive index $n=1.45$, the nonlinear refractive index $n_{2}=3.2 \cdot 10^{-20} \mathrm{~m}^{2} / \mathrm{W}$, and the fiber core radius $r=4 \cdot 10^{-6} \mathrm{~m}$. The NOLM transfer characteristics are presented as functions of the peak power of an individual control pulse, whereas the average control-beam power is considered constant in all cases and equal to $0.7 \mathrm{~W}$.

First let us study the possibility of influencing the NOLM switching by setting the control-beam peak power and the angle of polarization between the linearly polarized input $\mathrm{CW}$ data and control beam. The polarizations of the control and CCW data beams are kept parallel in all cases. The numerical results show that the NOLM transmission is periodically dependent on the control-beam peak power (Fig. 2). Moreover, without changing the control-beam peak power, transmissions vary in a wide range by changing the mutual angles of polarization between input data and control beams. We refer to the value of the controlbeam power at which the first maximum transmittance occurs as the critical power. That means that the critical power is the power necessary to switch the NOLM from the minimum to the maximum transmission. For increasing mutual angles of polarizations between the input data and control beams from $0 \mathrm{deg}$ to $90 \mathrm{deg}$, the values of the critical power increase approximately three times (from

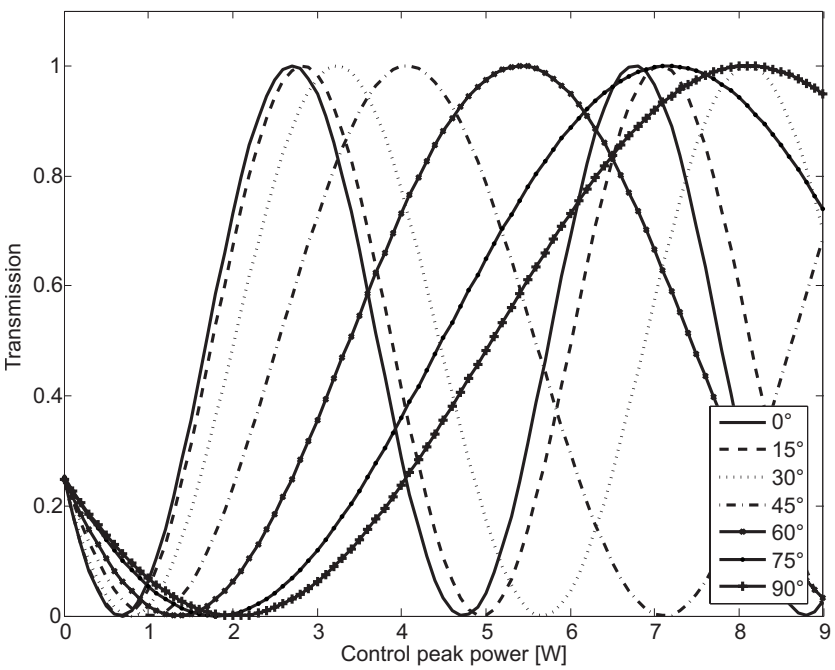

(a)

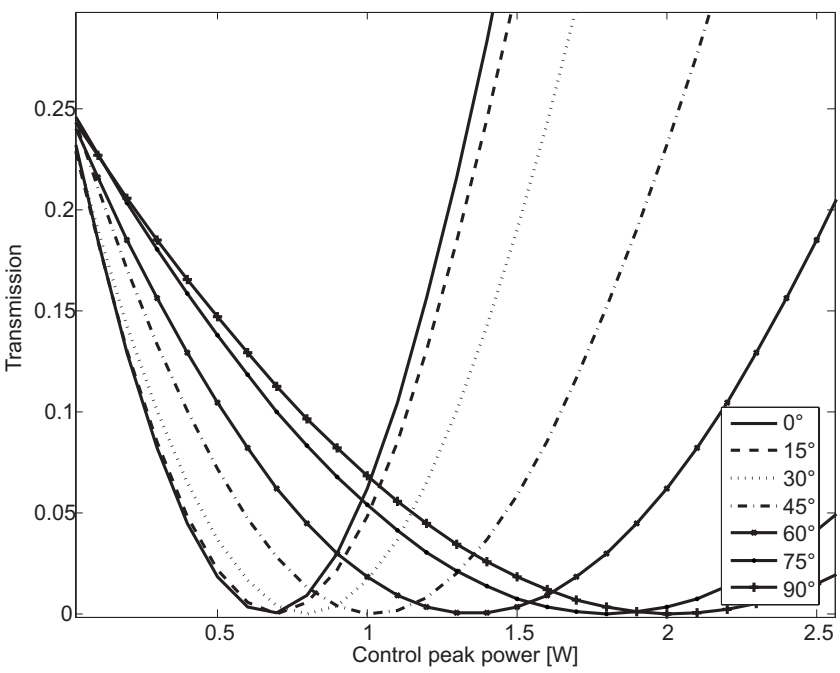

(b)

Fig. 2 (a) Transmission of the NOLM versus the peak control-beam power and the mutual angles of polarization between the linearly polarized input CW data and control beam. (b) Zooms in on a part of Fig. 2(a). 


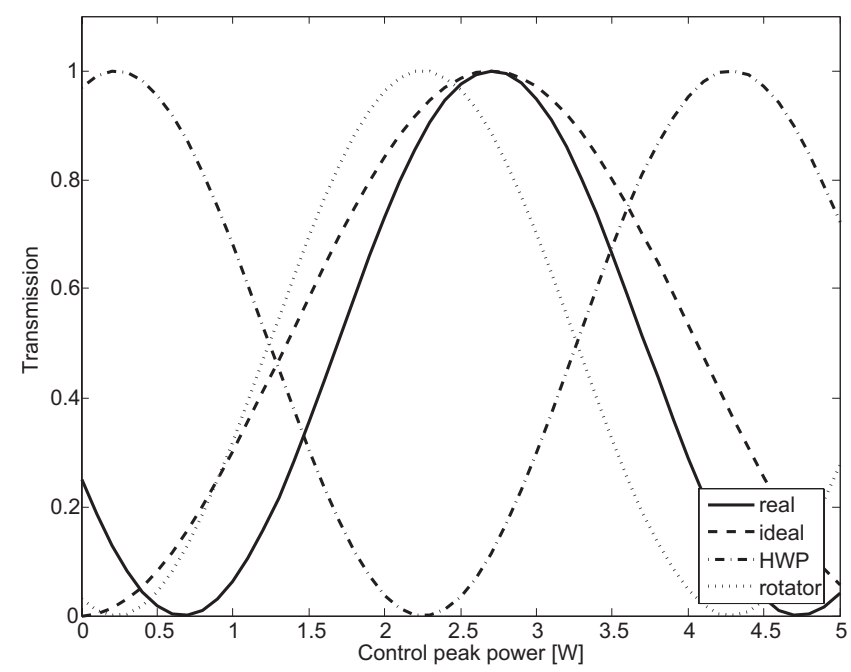

(a)

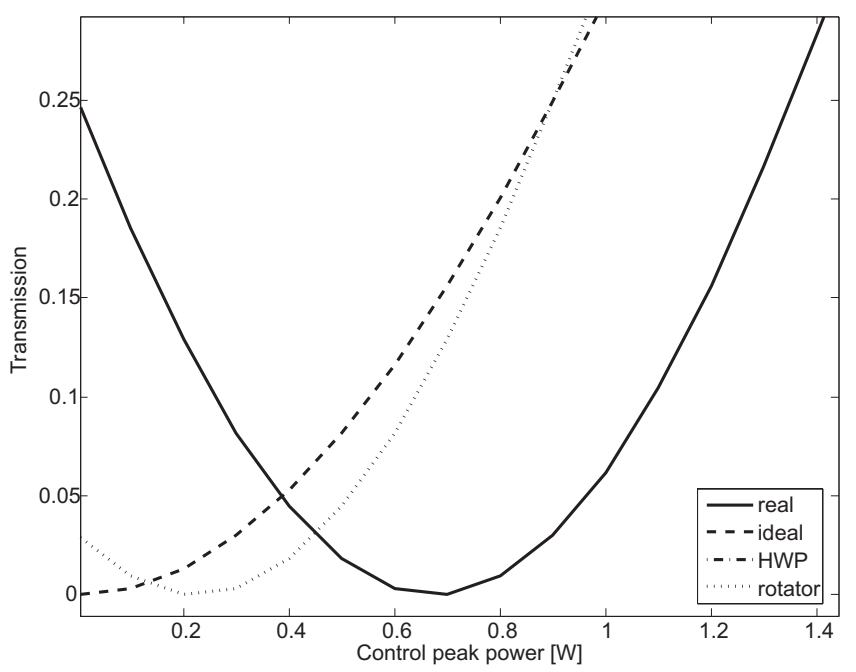

(b)

Fig. 3 (a) Transmission of the NOLM as a function of the control-beam peak power for four different simulation schemes. HWP_a half-wave plate. (b) Zooms in on a part of Fig. 3(a).

$\sim 2.8 \mathrm{~W}$ at $0 \mathrm{deg}$ to $\sim 8.2 \mathrm{~W}$ at $90 \mathrm{deg}$ ). This implies that the critical power and the polarization angle can be adjusted by the polarization to achieve the optimum switching. In addition, the control-beam peak power window between two adjacent transmittance extremes increases with the increasing angle of polarizations, being $\sim 0.7 \mathrm{~W}$ for polarization angles of $0 \mathrm{deg}$ and $30 \mathrm{deg}$, yet $\sim 2.6 \mathrm{~W}$ for polarization angles of $60 \mathrm{deg}$ and $90 \mathrm{deg}$.

Figure 3 displays the transmission characteristics as a function of the peak power of the control beam. The control and data beams are linearly polarized, and the mutual angle between their polarizations is equal to zero.

The transmission simulated here represents not only NOLM transfer properties, but is also a figure of merit of the interchannel cross talk between demultiplexed and nondemultiplexed channels. The main reason for the cross talk is the XPM-induced phase shift. The first curve represents the ideal case, i.e., if the transmission is zero at the zero control-beam peak power. That means that no cross talk is observed between the demultiplexed and nondemultiplexed OTDM channels at the output. But in practice, even if the control peak power is zero, the nonzero transmission occurs, as illustrated in Fig. 3. In this case, referred to as real in Fig. 3, the transmission of $\sim 25 \%$ occurs even if the control-beam peak power equals zero. This is due to a small XPM effect depending not on the instantaneous but on the average control-beam power causing the cross talk between the demultiplexed and nondemultiplexed channel.

The next course of simulations proves that the variety of the adjustment of transmission characteristics, including the cross-talk reduction, can be extended by using a half-wave plate or a rotator in the loop. The curve in Fig. 3 referred to as HWP represents the case of using a half-wave plate (HWP) placed close to the coupler of the NOLM (in the CCW direction) in terms of Fig. 1. This device allows rotating the polarization of the data-beam fraction propagating in the CCW direction by an optional angle. Then an additional phase shift occurs, causing the shift of transmission characteristics. The angle of the HWP was adjusted so that the polarization of the data-beam fraction, propagating in the CCW direction, was set perpendicular to the polarization of the control beam. As Fig. 3 shows, at the zero control-beam peak power, the NOLM becomes almost fully transparent (transmission of $\sim 100 \%$ ). However, the transmission of $\sim 0$ is necessary for the crosstalk reduction (the ideal curve, Fig. 3). Therefore, the transmission characteristic should be shifted back to the initial position. This can be achieved by using a cascade of several HWPs. A rotator placed in the loop can play the role of an HWP cascade. The rotator works like an HWP; the difference is that no additional phase shift occurs. Figure 3 represents also the NOLM transmission by using the rotator. The angle of the rotator is set to $90 \mathrm{deg}$, i.e., the polarizations of the databeam fraction propagating in the $\mathrm{CCW}$ direction and the control beam are set perpendicular. The zoomed part [Fig. $3(\mathrm{~b})]$ shows that the cross talk can be strongly reduced (to $\sim 3 \%$ ) by applying the rotator.

Figure 4 illustrates the switching by setting the controlbeam peak power and the angle of polarization between the linearly polarized input data and control beam in the NOLM with a rotator placed in the loop. The angle of the rotator is set to $90 \mathrm{deg}$, the polarization angle of the input data beam equals zero, the polarization angle of the input control beam is between $0 \mathrm{deg}$ and $90 \mathrm{deg}$. The other parameters are kept the same as in the previous simulations. The control beam is here again to achieve a switching depending periodically on the input beam power. However, in this case, a different effect on the interchannel cross-talk reduction occurs depending on the polarization angle of the input control beam. As can be seen from the zoomed-in image in Fig. 4(b), the interchannel cross talk is reduced to a greater extent when the polarization angle of both data and control beams is equal to zero (meaning that in the $\mathrm{CW}$ direction, the polarizations of the two beams are parallel to each other, while in the $\mathrm{CCW}$ direction, they are perpendicular). The increase of the input control-beam polarization angle causes the damped efficiency of the cross-talk reduction. From the simulations, we deduce that a signifi- 


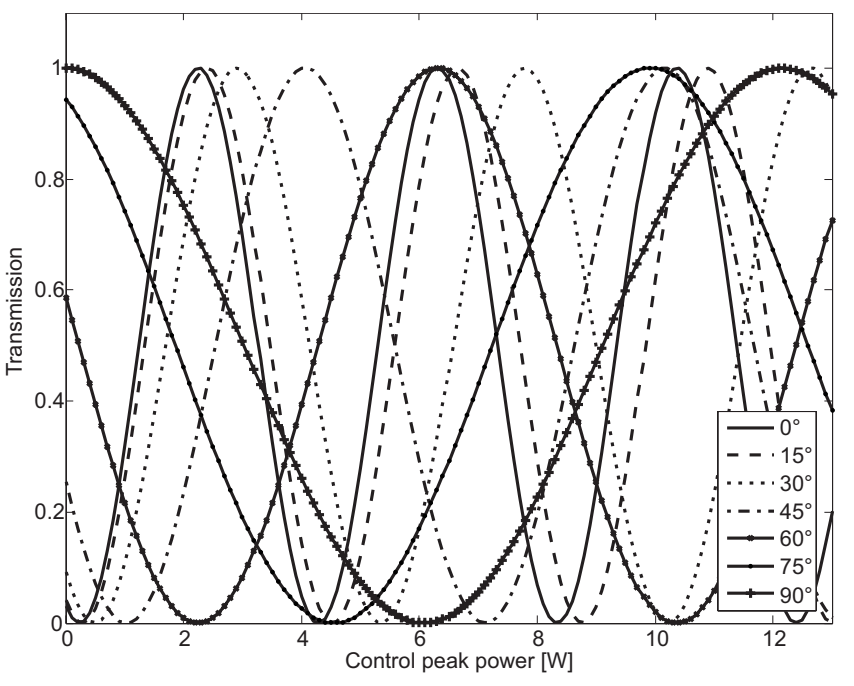

(b)

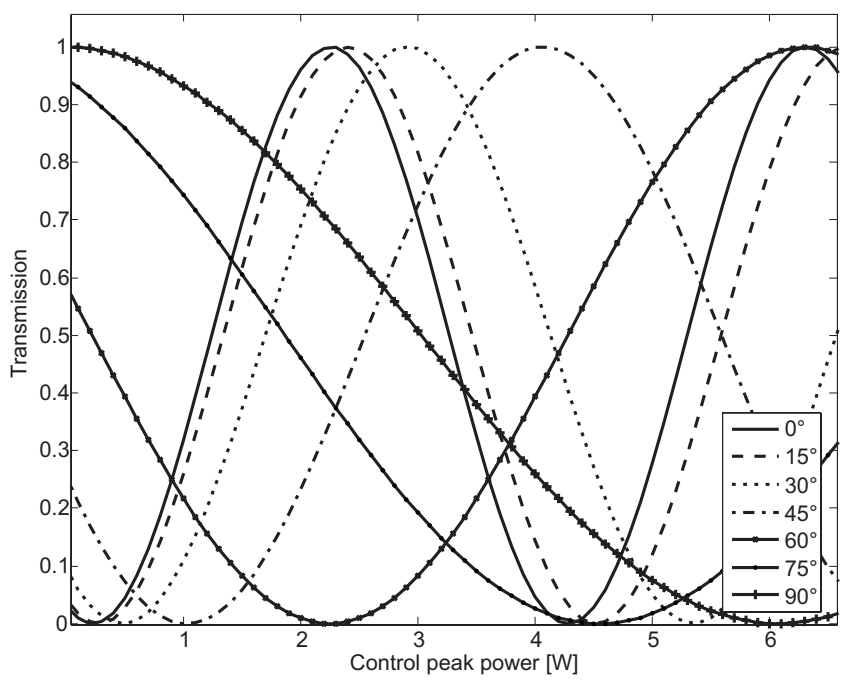

(b)

Fig. 4 (a) Transmission of the NOLM, including the rotator versus the peak control-beam power and the mutual angles of polarization between the linearly polarized input data and control beam. (b) Zooms in on a part of Fig. 4(a).

cant cross-talk minimization can be achieved at angles smaller than $30 \mathrm{deg}$. If the polarization angle is $\geqslant 45 \mathrm{deg}$, it is better for cross-talk reduction to use the configuration without the rotator in the loop. The maximum cross talk will be reached when the polarization angle equals $90 \mathrm{deg}$. (In the $\mathrm{CW}$ direction, the polarizations of the two beams are set perpendicular to each other, while in the $\mathrm{CCW}$ direction, they are set parallel.)

As we can see, the mutual angles between polarization components of the input data and control beams influence very sensitively the cross talk between the demultiplexed and nondemultiplexed channels. According to the numerical results, we conclude that this sensitivity can be degraded by an adaptive adjustment of the rotator angle according to the polarization angle of the input data and control beams.

As we have shown, the power exchanging process occurring between the data and control beams influences the NOLM transmission limits. From all numerical results, one can clearly see the transfer from the polarization and peak power changes of the control beam to the NOLM transmission, i.e., to the data beam. This is evidence that the NOLM of our scheme works similarly to schemes as in Refs. 2 and 17 but with a reduced number of optical elements necessary for OTDM demultiplexing in the usual optical telecommunication band of $\sim 1550 \mathrm{~nm}$.

\section{Conclusions}

In this paper, the performance of a control-beam-driven NOLM of a new design is simulated. In the presented NOLM scheme, a birefringent twisted fiber and a symmetrical coupler are supposed. Results of our theoretical model and numerical simulations indicate that the full switching operation can be achieved by the control-beam power and the polarization orientation only. The critical power necessary to switch the NOLM from the minimum to maximum transmission can also be adjusted through the angle change between the input data- and control-beam po- larizations. Furthermore, attention is focused on the cross talk between demultiplexed and nondemultiplexed beams as the main parameter for the switching function of an NOLM in OTDM systems. Such operation is very promising and should be implemented as a subsystem for the advanced so-called all-optical communication systems and networks. The numerical results show that the effect of the cross talk between the demultiplexed and nondemultiplexed OTDM channels in the NOLM as an OTDM demultiplexer can be strongly reduced (up to 8 times) through the management of the relative polarization orientations between co- and counterpropagating data and control beams.

\section{Acknowledgments}

This work was based on research activities supported by the Slovak Research and Development Agency under Project APVV COST-0041-06 and the COST 291 Action titled "Towards Digital Optical Networks." D. Grendár started this work during his study stay at the Faculté Polytechnique de Mons (Belgium) within the framework of the Socrates-Erasmus Program in 2005 under the supervision of Prof. Michel Blondel and Dr. Olivier Pottiez. They both are greatly acknowledged for this opportunity and fruitful discussions.

\section{References}

1. N. J. Doran and D. Wood, "Nonlinear optical loop mirror," Opt. Lett. 13, 56-58 (1988).

2. A. Bogoni, M. Scaffardi, P. Ghelfi, and L. Poti, "Nonlinear optical loop mirrors: investigation solution and experimental validation for undesirable counterpropagating effects in all-optical signal processing," IEEE J. Sel. Top. Ouantum Electron. 10, 1115-1123 (2004).

3. B. E. Olsson and P. A. Andrekson, "Polarization independent demultiplexing in a polarization diversity nonlinear optical loop mirror,' IEEE Photon. Technol. Lett. 9, 764-766 (1997).

4. D. Wang, E. A. Golovchenko, A. N. Pilipetskii, C. R. Menyuk, and M. F. Arend, "Nonlinear optical loop mirror based on standard communication fiber," J. Lightwave Technol. 15, 642-646 (1997).

5. M. F. Arend, M. L. Dennis, I. N. Duling III, E. A. Golovchenko, A. N. Pilipetskii, and C. R. Menyuk, "Nonlinear-optical loop mirror demultiplexer using a random birefringence fiber: comparisons between simulations and experiments," Opt. Lett. 22, 886-888 (1997). 
6. H. Sotobayashi, Ch. Sawaguchi, Y. Koyamada, and W. Chujo, "Ultrafast walk-off-free nonlinear optical loop mirror by a simplified configuration for $320 \mathrm{Gbit} / \mathrm{s}$ time-division multiplexing signal demultiplexing," Opt. Lett. 27, 1555-1557 (2002).

7. J. D. Moores, K. Bergman, H. A. Haus, and E. P. Ippen, "Optical switching using fiber ring reflectors," J. Opt. Soc. Am. B 8, 594-601 (1991)

8. H. C. Lim, F. Futami, and K. Kikuchi, "Polarization independent wavelength-shift-free optical phase conjugator using anonlinear fiber Sagnac interferometer," IEEE Photon. Technol. Lett. 11, 578-580 (1999).

9. W. W. Tang, C. Shu, and K. L. Lee, "Rational harmonic mode locking of an optically triggered fiber laser incorporating a nonlinear optical loop modulator," IEEE Photon. Technol. Lett. 13, 16-18 (2001).

10. I. N. Duling III, "All-fiber ring soliton laser mode locked with anonlinear mirror," Opt. Lett. 16, 539-541 (1991).

11. M. D. Pelusi, Y. Matsui, and A. Suzuki, "Pedestal suppression from compressed femtosecond pulses using anonlinear fiber loop mirror," IEEE J. Quantum Electron. 35, 867-874 (1999).

12. M. Attygalle, A. Nirmalathas, and H. F. Liu, "Novel technique for reduction of amplitude modulation of pulse trains generated by subharmonic synchronous mode-locked laser," IEEE Photon. Technol. Lett. 14, 543-545 (2002)

13. A. Bogoni, P. Ghelfi, M. Scaffardi, and L. Poti, "All-optical regeneration and demultiplexing for $160-\mathrm{Gb} / \mathrm{s}$ transmission systems using a NOLM-based three-stage scheme," IEEE J. Sel. Top. Quantum Electron. 10, 192-196 (2004)

14. H. Shi and J. Lin, "Theoretical analysis on polarization deviation and switch window optimization in nonlinear optical loop mirror demultiplexer," J. Lightwave Technol. 17, 2572-2576 (1999).

15. K. Uchiyama, H. Takara, T. Morioka, S. Kawanishi, and M. Saruwatari, "Effects of control-signal pulse walk-off on BER performance of nonlinear optical loop mirror demultiplexer," Electron. Lett. 29 1313-1314 (1993)

16. E. A. Kuzin, N. Korneev, J. W. Haus, and B. Ibarra-Escamilla, "Theory of nonlinear loop mirrors with twisted low-birefringence fiber," J. Opt. Soc. Am. B 18, 919-925 (2001).

17. B. Ibarra-Escamilla, E. A. Kuzin, O. Pottiez, J. W. Haus, F. GutierrezZainos, R. Grajales-Coutiño, and P. Zaca-Moran, "Fiber optical loop mirror with a symmetrical coupler and a quarter-wave retarder plate in the loop," Opt. Commun. 242, 191-197 (2004).

18. B. Ibarra-Escamilla, E. A. Kuzin, P. Zaca-Morán, R. GrajalesCoutiño, F. Mendez-Martínez, O. Pottiez, R. Rojas-Laguna, and J. W. Haus, "Experimental investigation of the nonlinear optical loop mirror with twisted fiber and birefringence bias," Opt. Express 13 10760-10767 (2005)

19. O. Pottiez, E. A. Kuzin, B. Ibarra-Escamilla, F. Gutiérrez-Zainos, U. Ruiz-Corona, and J. T. Camas-Anzueto, "High-order amplitude regularization of an optical pulse train using a power-symmetric NOLM with adjustable contrast," IEEE Photon. Technol. Lett. 17, 154-156 (2005).

20. O. Pottiez, E. A. Kuzin, B. Ibarra-Escamilla, J. T. Camas-Anzueto, and F. Gutiérrez-Zainos, "Easily tunable nonlinear optical loop mirror based on polarization asymmetry," Opt. Express 12, 3878-3887 (2004).

21. S. F. Feldman, D. A. Weinberger, and H. G. Winful, "Polarization instability in atwisted birefringent optical fiber," J. Opt. Soc. Am. B 10, 1191-1201 (1993).

22. T. Tanemura and K. Kikuchi, "Circular-birefringence fiber for nonlinear optical signal processing," J. Lightwave Technol. 24, 4108 4119 (2006)

23. G. P. Agrawal, Nonlinear Fiber Optics, Academic Press, London (2001)

24. S. Kumar, A. Selvarajan, and G. V. Anand, "Nonlinear copropagation of two optical pulses of different frequencies in birefringent fibers," J. Opt. Soc. Am. B 11, 810-817 (1994).

25. E. A. Kuzin, J. M. Estudillo-Ayala, B. Ibarra-Escamilla, and J. W. Haus, "Measurements of beat length in short low-birefringence fibers," Opt. Lett. 26, 1134-1136 (2001).

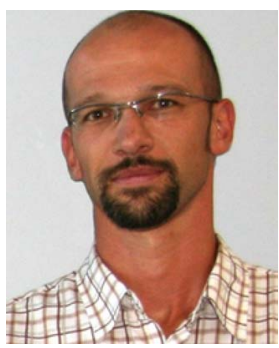

Drahomír Grendár received the telecommunication engineering degree from the University of Žilina, Slovakia, in 2002. From 2002 to 2005 , he was a graduate student in the Department of Telecommunications at the University of Žilina. He studied for the $\mathrm{PhD}$ degree in the field of optical time division multiplexing (OTDM), focusing specifically on the theoretical and numerical investigation of nonlinear optical loop mirrors for OTDM applications. In 2005, he received an internship in the European Socrates/Erasmus Program at the Fac- ulté Polytechnique de Mons, Belgium, where he cooperated with Olivier Pottiez in the area of OTDM systems. He currently works in Slovak Telecom as a product manager.

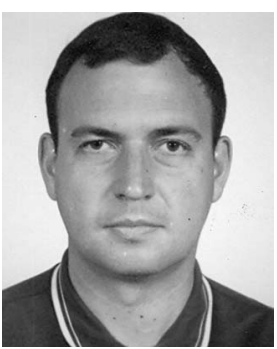

Olivier Pottiez received the electrical engineering and $\mathrm{PhD}$ degrees from the Faculté Polytechnique de Mons, Belgium, in 1997 and 2001, respectively. In 2003, he pursued a postdoctoral stay at the Instituto Nacional de Astrofísica, Óptica y Electrónica (INAOE), Puebla, Mexico. He is currently a researcher in the Fiber Optics Department of the Centro de Investigaciones en Óptica (CIO), León, Mexico. His present research interests include mode-locked fiber lasers for ultrashort pulse generation and fiber Sagnac interferometers for ultrafast photonics applications.

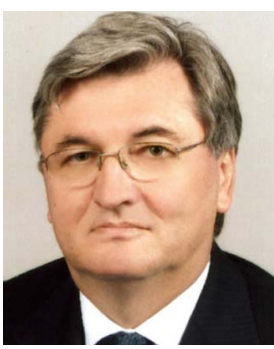

Milan Dado is full professor in the Department of Telecommunications and Multimedia at the Faculty of Electrical Engineering, University of Žilina. His research and education activities are focused on different areas of information and communication technologies and services in general. In the field of optical communications, he has investigated different topics in ultra-high-capacity optical transmissions networks, characterization of advanced fibers for new photonic networks, measurement techniques for active and passive fibers, and new all-optical IP transparent optical networks. In these fields, he has mainly investigated systems with the aid of numerical computer modeling.

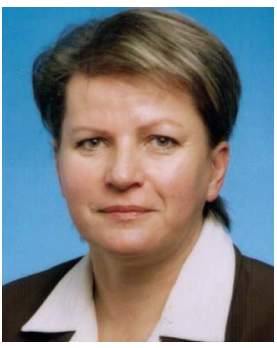

Jarmila Müllerová received her MS degree in experimental physics and her PhD in quantum electronics and nonlinear optics from Comenius University, Bratislava, Slovakia. Since 2003, she has been with the University of Žilina, Slovakia. Currently, she is an associate professor in telecommunications at the Department of Engineering Fundamentals, the Faculty of Electrical Engineering. Her ongoing research interests include optical properties of solids, thin films optics, and optics of multilayered structures and interfaces. Her attention is also focused on wavelength-sensitive devices for the physical layer of all-optical communication networks, namely for WDM and OTDM applications.

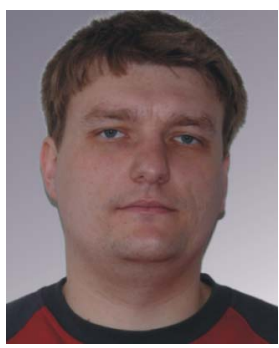

Jozef Dubovan received a telecommunication engineering degree from the University of Žilina, Slovakia, in 2005. Since 2005, he has been a PhD student in the Department of Telecommunications at the University of Žlina. His PhD thesis is oriented on research and investigation of full-transparent optical networks and optical switches. In 2007, he received an internship at the Alcatel-Lucent Deutschland in Stuttgart and developed the numerical models for evaluation of the time dynamics of installed fibers' PMD property. 